\title{
Coordinated Corrective Control for Transient Stability Enhancement in Future Great Britain Transmission System
}

\author{
Inmaculada Martínez Sanz, Balarko Chaudhuri, Goran Strbac \\ Department of Electrical and Electronic Engineering \\ Imperial College London \\ London, UK \\ \{im1309, b.chaudhuri, g.strbac\}@imperial.ac.uk
}

\begin{abstract}
This paper demonstrates a corrective control strategy through fast actuators (TCSC FACTS and HVDC links, both LCC and VSC) in order to enhance the transient stability in the future Great Britain (GB) transmission network. A model predictive control (MPC) scheme that relies on system wide-area measurements is employed for coordinated control action through these power electronic devices with the aim of preserving the system stability without having to constrain prefault transfer levels. Case studies employing detailed dynamic models are presented to demonstrate the effectiveness of the proposed approach for different formulations on a representative equivalent model of the future GB transmission grid.
\end{abstract}

Index Terms-Corrective control, HVDC transmission, model predictive control (MPC), TCSC, transient stability.

\section{INTRODUCTION}

Significant increase in power transfer capacity between Scotland and England/Wales would be required to ensure security of supply with high wind penetration in the future Great Britain (GB) transmission system [1]. To that end, National Grid, the GB system operator, has planned two subsea HVDC cable links, together with series compensation (some of which could be TCSCs) to increase the transfer capacity of the existing two $400 \mathrm{kV}$ AC double-circuit lines that form the connection between Scotland and England [2]. Alongside increased capacity, corrective control through the planned reinforcements could be used to enhance the system transient stability and, thereby, allow higher pre-fault loading of the existing AC corridors without compromising security. Such corrective control actions allow more time for optimized decisions (e.g. generators re-scheduling) after the outage of one of the double-circuit lines.

In this paper, a model predictive control (MPC) scheme is presented for corrective action through the planned network reinforcements to address the angle stability issues present in the GB grid. MPC is adopted because it can explicitly account for system constraints and changing operating conditions in a multi-variable framework [3]. Therefore, it is suited for

National Grid support to I. Martínez Sanz is gratefully acknowledged. coordinated control of power electronic actuators with limited short-term overload capability.

Although challenging from a computational point of view, recent papers have investigated the application of MPC for transient stability improvement. In [4] several stabilizing actions (generation tripping, load shedding and series capacitance) are considered. In [5] the focus is on robust first swing stability protection using FACTS devices and [6] is about adjusting the generators mechanical power. Application of MPC to HVDC control to solve loss of synchronism problems was first proposed in [7] using a single LCC-HVDC link considering simplified system dynamics. Use of MPC with VSC-HVDC links was introduced in [8] and later extended in [9] to damp oscillations in a large AC system. However, MPCbased corrective control through HVDC links, VSC-HVDC in particular, has not been explored in detail to solve first swing instability problems.

The contribution of this paper is the application of MPC for coordinated corrective control action through different fast actuators with distinct behaviour (TCSCs, LCC and VSC converters) under a few representative scenarios in the future GB transmission system. The corrective control initially presented in [10]-[11] using a single LCC-HVCC actuator is extended here to a multi-actuator context. Effect of using different cost functions on the system dynamic response is also presented.

\section{Corrective Control in Future Great Britain TRANSMISSION SYSTEM}

\section{A. The Anglo-Scottish Boundary}

In the GB transmission network the interconnection between Scotland and England (also known as 'Anglo-Scottish boundary' or 'B6 boundary') has been identified as a critical transmission corridor. The transfer capacity is limited by angle stability considerations and is inadequate to support the projected growth in power transfer from the North to the South of the UK, primarily onshore and offshore wind [1]. Currently the transfer limit is set by the N-2 security criterion and possible contribution from corrective control is not considered [12]. The Electricity Networks Strategy Group (ENSG) published a report in 2009 (and updated in 2012) on 
the strategic reinforcements required to facilitate connection of the new generation mix to the GB transmission network by 2020 [2]. The main proposed transmission reinforcements to increase the B6 boundary capability are the installation of series and shunt compensation around the Scotland-England interconnection and two sub-sea HVDC links embedded in the GB synchronous power system, along the west and east coast, as shown in Fig. 1. The links are likely to be based on different converter technology and are also referred as Western LCCHVDC link (project approved and order placed) and Eastern VSC-HVDC link (status still unclear), respectively.

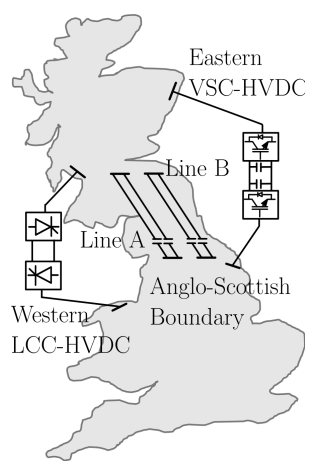

Figure 1. Planned reinforcements in the GB transmission network.

It is expected that the installation of series compensation will allow each double-circuit AC corridor to be operated close to its thermal capacity of $4.4 \mathrm{GW}$. In addition, the Western LCC-HVDC link would add 2.2 GW capacity [13] and the Eastern VSC-HVDC link could be around $2 \mathrm{GW}$ and likely to form the backbone of an integrated offshore network.

\section{B. Three-Machine GB Equivalent Test System}

A test system representative of the GB transmission network was set up in DIgSILENT PowerFactory to evaluate the proposed coordinated control strategy. This model is based on the one described in [14] and shown in Fig. 2. Its structure and parameters have been chosen to be roughly representative of the English-Scottish power system. It consists of three generation areas: Area 1, corresponding to the Southern Scotland network (owned by Scottish Power Transmission Limited SPT), Area 2 to the Northern Scotland network (owned by Scottish Hydro Electric Transmission plc - SHELT), and Area 3 to the England and Wales network (owned by National Grid Electricity Transmission plc - NG). These three entities own the GB onshore transmission network, which is operated by NG. In the test system, Areas 1 and 2 are connected to the main system, Area 3, by two double circuit parallel tie-lines, Line A and Line B, representing the Anglo-Scottish boundary. Area 1 is closely coupled to the main system, while Area 2 is relatively remote.

An aggregated round rotor synchronous generator is considered to represent each area of the system. The three generators are equipped with excitation control, local PSS and are driven by steam turbines with governor control, (see [14] for details). This three-machine GB equivalent test system has been

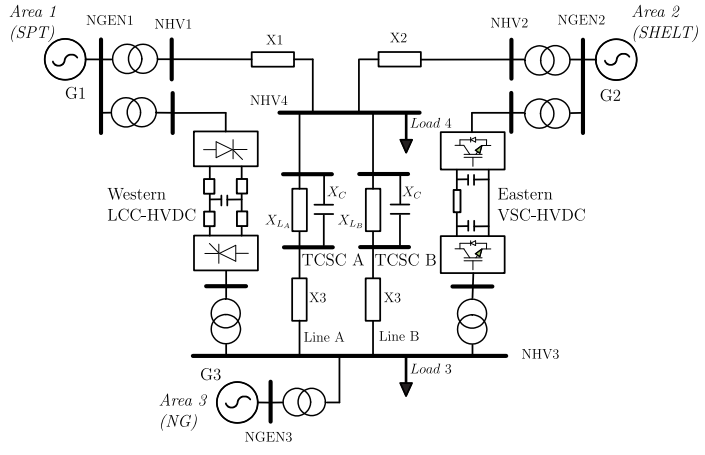

Figure 2. Three-machine GB equivalent test system.

adapted to accommodate the expected network reinforcements in the system, as seen in Fig. 2. The operating condition investigated in the studies presented in this work slightly differs from the one in [14]. Stressed operation of the system is considered in the system with $4.4 \mathrm{GW}$ going into the main system through the two tie-lines. Generation levels in Area 1 and 2 were doubled and Load 4 was adjusted to achieve this.

Despite its simplicity, this three-machine network is a reasonable representation of the dynamic behaviour of the GB transmission system and, in particular, the problems associated with high power transfer across the Anglo-Scottish boundary. A detailed model of the GB transmission system developed by SPT [15] exhibited a sharp transition from a stable and well damped to transiently (first swing) unstable condition after the outage of one of the two transmission corridors for high prefault power transfers through these tie-lines. This behaviour was reproduced in this small test system.

\section{DynAmic MOdel of FAST ACTUATORS}

This section briefly discusses the modeling and control of the three key reinforcements incorporated to the three-machine GB equivalent test system. As the number of fast actuators increase in the system, coordinated control would be desirable to provide effective support to the AC grid.

\section{A. Thyristor Controlled Series Capacitor (TCSC)}

A thyristor controlled series capacitor (TCSC) is a combination of a fixed capacitor bank connected in parallel with a thyristor controlled reactance (TCR). It provides smooth variation of a transmission line series reactance allowing to control the power flowing through it [16]. The dynamic characteristics of the TCSC have been approximated using a fixed capacitor $\left(X_{C}\right)$ in parallel with a variable reactance $\left(X_{L}\right)$. A single time constant $T$ is used to model the control response of the TCSC to track a specified $X_{L}^{*}$. Two TCSCs (TCSC A and TCSC B) are included in the three-machine GB equivalent test system, on Line $A$ and on Line B. The following parameters were used: $X_{C}=10 \Omega, X_{L_{A}}=X_{L_{B}}=26.99 \Omega, X_{3}=45.38 \Omega$ and $T=0.05 \mathrm{~s}$.

\section{B. LCC-HVDC Converter}

The LCC converter is built with thryristor valves and relies on the AC system voltage for commutation. The model of the 
LCC converters is summarized in the following expressions of the DC voltages $V_{d c}$ and currents $I_{d c}$ at the rectifier and inverter terminals [17]:

$$
\begin{aligned}
V_{d c}^{r e c} & =\frac{3 \sqrt{2}}{\pi} V_{L L} \cos \left(\alpha^{r e c}\right)-R_{c} I_{d c}^{r e c} \\
V_{d c}^{i n v} & =\frac{3 \sqrt{2}}{\pi} V_{L L} \cos \left(\pi-\alpha^{i n v}\right)+R_{c} I_{d c}^{i n v}
\end{aligned}
$$

where $V_{L L}$ is the $\mathrm{AC}$ system line-to-line voltage, $\alpha^{r e c}$ and $\alpha^{i n v}$ are the rectifier and inverter firing angles and $R_{c}$ is the commutating resistance which accounts for the voltage drop reduction due to the commutation overlap $(\mu)$.

The simplified control structure of the converters is shown in Fig. 3. Under normal operation, the DC power $\left(P_{d c}^{*}\right)$ is regulated by the rectifier and the extinction angle $\gamma(\gamma=\pi-$ $\alpha-\mu$ ) by the inverter. For depressed voltage levels, a voltage dependant current order limiter (VDCOL) that controls current in proportion to voltage is considered.

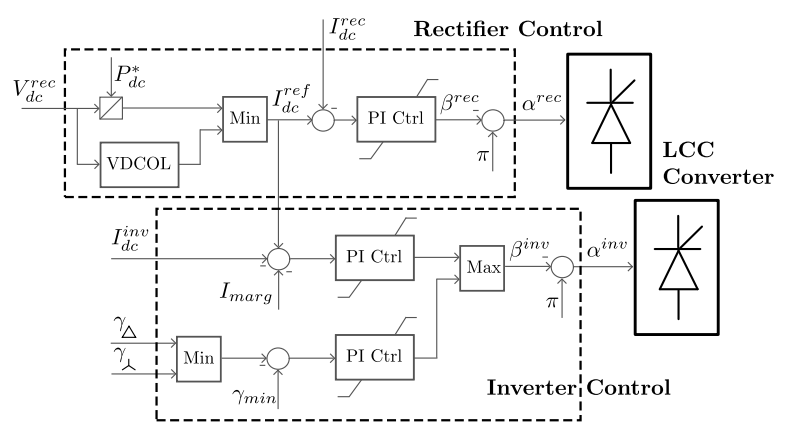

Figure 3. LCC-HVDC converters control.

The Western LCC-HVDC link integrated in the three machine GB equivalent test system has a power rating of $2.2 \mathrm{GW}$ and a voltage rating of $\pm 500 \mathrm{kV}$. In steady-state condition the LCC link is set to transfer $2.2 \mathrm{GW}$ from North to South. The data for the converters and filters are based on the CIGRè HVDC benchmark model [18], with the parameters adapted for the new ratings. The data used for each DC cable is: 500 $\mathrm{km}, R_{d c}=0.02 \Omega / \mathrm{km}, X_{d c}=0.2 \Omega / \mathrm{km}$ and smoothing reactor $L=0.5968 \mathrm{H}$.

\section{VSC-HVDC Converter}

The VSC converters are based on IGBT switches, which allows independent control of both active and reactive power injections at the terminal buses. The converter model equations can be expressed in terms of the converter AC current components $\left(i_{d}, i_{q}\right)$ in a $d q$ reference frame [19]:

$$
\begin{aligned}
V_{t_{d}}-V_{s_{d}} & =R_{c} i_{d}-w L_{c} i_{q}+L_{c} \frac{d i_{d}}{d t} \\
V_{t_{q}} & =R_{c} i_{d}-w L_{c} i_{q}+L_{c} \frac{d i_{d}}{d t}
\end{aligned}
$$

where $R_{c}$ and $L_{c}$ are the resistance and inductance of the phase reactor that connects the converter to the AC grid. $V_{s}$ is the voltage at the connection point and $V_{t}$ is the converter terminal voltage.
The vector control strategy usually applied for VSC-HVDC schemes is shown in Fig. 4. The converter is controlled by tracking the current injected into the AC network. The control associated to each converter includes two PI controllers in cascade. The outer slower loop defines the $d$ and $q$ current reference values and the internal fast loop controls these currents by adjusting the the converter terminal voltage.

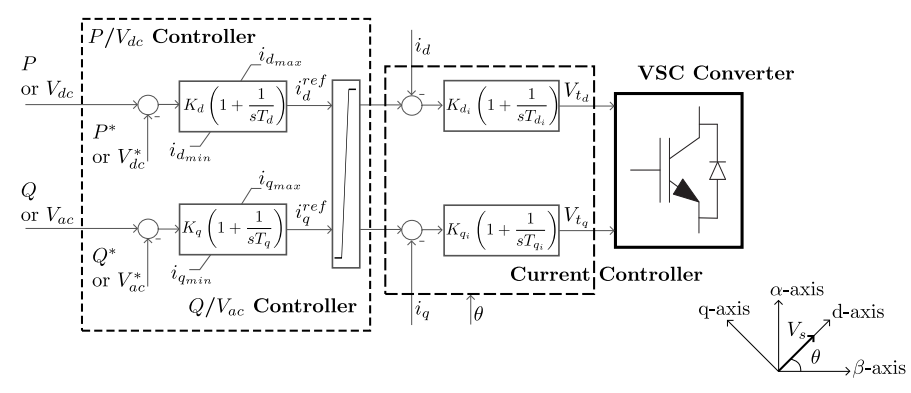

(a)

(b)

Figure 4. VSC-HVDC converter vector control strategy; (b) $d q$ frame for decoupled control.

In the active control loop for the VSC-HVDC system, one converter is set to control the active power (rectifier) and the other must be set to control the DC link voltage (inverter), adjusting its power flow. The reactive power control of the two converters is independent and each converter can control either the reactive power or the voltage on the AC side [20].

The Eastern VSC-HVDC link embedded in the threemachine GB equivalent test system is modeled as a $2 \mathrm{GW}$ $\pm 500 \mathrm{kV}$ link with the following parameters: $R_{c}=0$, $u_{k}=15 \%$ (short circuit impedance), $C_{c}=76 \mu F$ (DC capacitors). The DC line is assumed to be $400 \mathrm{~km}$ long.

\section{MPC CONTROLlER DESIGN}

This work focuses on rotor-angle stability, which quickly evolve in the seconds time-frame and require a fast response. The objective of the proposed controller is to adjust the AC lines series compensation and HVDC links power orders to improve the post-fault stability of the system (i.e. corrective control). The controller relies on wide area measurements monitored across the system to obtain wider observability. Model predictive control (MPC) is adopted as it can explicitly handle control constraints, which are of particular importance for power electronic actuators with limited overload capability. Moreover, MPC allows coordinated control design in a multiinput multi-output (MIMO) framework which can ensure optimality of the control action with appropriate allocation of the control duty. An overview of the closed loop control structure is presented in Fig. 5.

MPC is a discrete time control strategy that uses a receding horizon approach [3]. At each time step $k$, the MPC controller solves the quadratic programming (QP) problem in (5) where $x \in \mathbb{R}^{n}$ denotes the system state variables, $u \in \mathbb{R}^{n_{u}}$ the system inputs and $y \in \mathbb{R}^{n_{y}}$ the system outputs, being $y_{\text {err }}(k)=y(k)-y_{\text {ref }}(k)$. 


$$
\begin{array}{ll}
\underset{\tilde{u}_{k}, \ldots, \tilde{u}_{k+N_{c}-1}}{\operatorname{argmin}} \sum_{i=0}^{N_{p}-1}\left(y_{\text {err }}(k+i+1)\right)^{T} Q\left(y_{\text {err }}(k+i+1)\right) & +\ldots+ \\
& +\ldots+\tilde{u}^{T}(k+i) R \tilde{u}(k+i)
\end{array}
$$

The input signals $u(k)$ are the reference set points of the controlled devices and the outputs $y(k)$ are different widearea measurements monitored across the system. Note that $\tilde{x}(k)=x(k)-x_{0}, \tilde{u}(k)=u(k)-u_{0}$ and $\tilde{y}(k)=y(k)-y_{0}$ when considering the initial operating point $\left(x_{0}, u_{0}, y_{0}\right)$. From the solution of this optimization problem, only the first step is actually applied to the system at time $k$. Then, the same process is repeated at the next time step $k+1$.

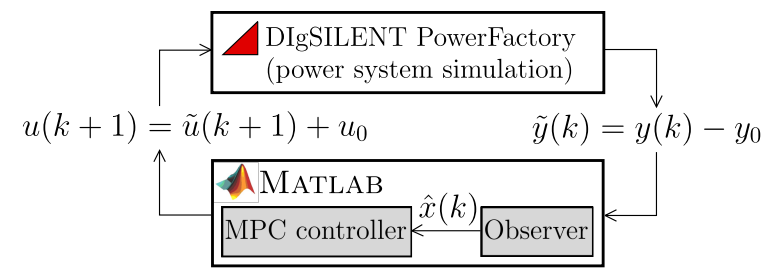

Figure 5. Overview of the MPC controller.

The next subsections cover the different aspects of the MPC formulation. Note that the controller is tested in ideal conditions (no communication delays, nor measurement errors). Future work should revisit these assumptions to evaluate their impact on the controller performance.

\section{A. Power System Model}

1) Linear Model Estimation: The MPC controller relies on a prediction model of the power system to determine the control actions that minimize the cost function in (5a). In this case, a discrete time linear state-space model is employed (5b)(5c). This model was obtained off-line through a subspace identification procedure, which provided a low-order blackbox model of the power system [21]. An overview of this identification routine is shown in Fig. 6. In steady state, the system is excited with low-energy level signals (typically pseudo random binary signals) and simulated responses are recorded. This data is processed (decimated, detrended and filtered) and the numerical algorithm n4sid in MATLAB is employed to identify a linear state-space model (matrices $A$, $B, C, D$ ) where the measured data has a good matching with the estimated model. The identified model is also validated against the response of the actual network to step pulses.

In the simulations presented next we consider that the power system model $(5 b)-(5 c)$ is updated after a topological change (e.g. line outage) by monitoring the breaker status. Following

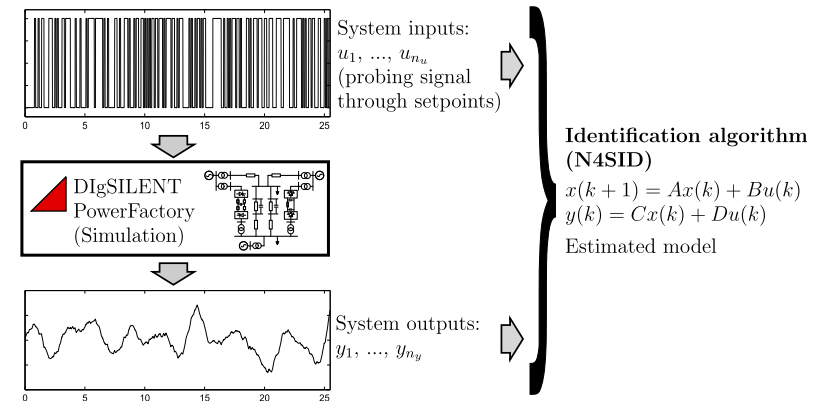

Figure 6. Overview of the power system identification procedure (off-line).

this approach, the excursion in the states is approximated by updating the discrete linear model. This updated model was also obtained off-line following the routine in Fig. 6 .

2) Observer Design: In order to calculate the predicted system states $\hat{x}(k)$ from the monitored system measurements $y(k)$ at each instant $k$, the controller is equipped with a linear state observer (Kalman filter with gain $L$ ). We assume that the system states are observable and no error nor delay in the estimator is initially considered.

\section{B. Cost Function}

Equation (5a) presents a typical quadratic cost function used in MPC problems [3]. The first term in (5a) penalizes the deviation of the predicted system outputs $y(k+i+1)$ from a reference trajectory $y_{r e f}(k+i+1)$ over a finite prediction horizon $N_{p}$. The second term minimizes the controller adjustments $\tilde{u}(k+1)$ from the initial operating point $u_{0}$ over a control horizon $N_{c}$ (where $N_{c} \leq N_{p}$ ). The diagonal matrices $Q$ and $R$ are the cost function weights, which are assumed to be time-invariant. Following standard assumptions of MPC, the matrices in the cost function are semipositive definite, with $Q \succeq 0$ and $R \succeq 0$.

The aim of the controller is to maintain the rotor angle stability of the system. Accordingly, different performance targets based on generator rotor angles and rotor speeds measurements that are directly related to this type of stability have been studied. The performance of different cost function formulations are compared in Section V.

\section{Control Constraints}

The MPC controller can explicitly account for constraints on the control variables as specified in (5e)-(5f), where $u_{H}$ and $y_{H}$ are the upper bounds and $u_{L}$ and $y_{L}$ are the lower bounds of the system inputs and outputs, respectively. In this work we consider limits on $u(k)$ according to the overload capabilities of the HVDC converters and the permissible line compensation level provided by a TCSC.

\section{CAse Study A: Corrective Control through WESTERN LCC-HVDC AND TCSC}

We initially demonstrate the effectiveness of a MPC-based corrective control strategy in the three-machine GB equivalent test system with only the TCSCs and the Western LCCHVDC link installed. Each TCSC provides a compensation 
level of $35 \%\left(X_{L_{A}}=X_{L_{B}}=26.99 \Omega\right)$ while the power transfer through the Western LCC-HVDC is $2.2 \mathrm{GW}$ in steady state. We consider a high loading scenario with $2.42 \mathrm{GW}$ being transferred through each tie-line of the Anglo-Scottish boundary. The contingency under study is the outage of one of these two double-circuit lines ( $\mathrm{N}-2$ security). At $t=1.0$ $\mathrm{s}$ a three-phase to ground fault occurs in Line $\mathrm{B}$, which is cleared after $80 \mathrm{~ms}$ by disconnecting the faulted line. For AC boundary transfers above $4.4 \mathrm{GW}$ the system is transiently unstable following the outage of Line B, if the Western LCCHVDC and the TCSCs are operated with a fixed reference setting. The red solid traces in Fig. 7 depict a more clear view of this phenomena. In Fig. 7 (a) and (b) the rotor angles of generators ${ }^{1} \mathrm{G} 1$ and G2 are seen to separate from the main system, loosing synchronism after the line trips.
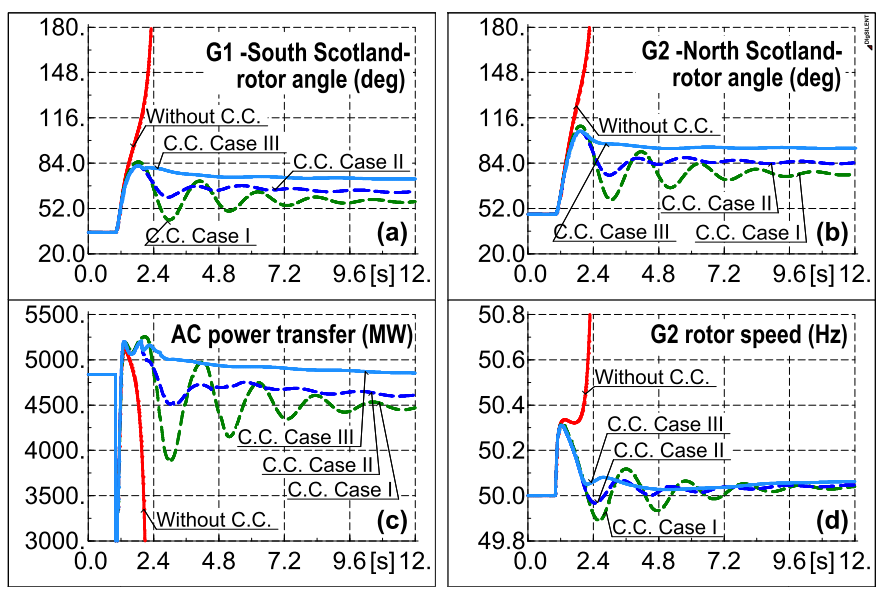

Figure 7. System dynamic response to Line B outage: (a) rotor angle $\theta_{G 1}$; (b) rotor angle $\theta_{G 2}$; (c) total Scotland-England AC boundary power transfer and (d) G2 rotor speed for the following scenarios: without C.C. (corrective control) in red solid traces; C.C. Case I in green dashed traces; C.C. Case II in blue dashed traces; C.C. Case III in light blue solid traces.

A MPC-based controller is designed next to stabilize the system, considering the LCC-HVDC power order $\left(P_{d c}^{*}\right)$ and the inductive reactance of the TCSC on Line A $\left(X_{L_{A}}^{*}\right)$ for corrective control action:

$$
u(k)=\left[P_{d c}^{*}(k) X_{L_{A}}^{*}(k)\right]^{T}
$$

The Western LCC-HVDC link is considered to have an overload capability of $15 \%$ (which could be sustained for tens of minutes [22]) and that the compensation level of TCSC A could be varied up to $40 \%$ :

$$
\left[\begin{array}{c}
1870 \mathrm{MW} \\
22.264 \Omega
\end{array}\right] \leq\left[\begin{array}{c}
P_{d c}^{*}(k) \\
X_{L_{A}}^{*}(k)
\end{array}\right] \leq\left[\begin{array}{c}
2530 \mathrm{MW} \\
26.99 \Omega
\end{array}\right]
$$

The response of the controller to various formulations using cost functions based on different wide-area measurements is analyzed next. Use of fixed and variable reference trajectories $\left(y_{r e f}\right.$ in (5a)) is also explored. In the simulations presented, the controller time step $T_{s}$ is $20 \mathrm{~ms}, N_{p}=25$ and $N_{c}=5$.

\footnotetext{
${ }^{1} \theta_{G i}$ is the relative rotor angle of generator $i$ based on the reference machine angle $\theta_{G 3}$.
}

\section{Case I. $\quad$ Fixed Rotor Angle Reference Trajectory}

First, the rotor angles of the generators are considered as measured outputs. The cost function is formulated to minimize the generator rotor angles deviation, $\theta_{G i}$, with respect to their pre-fault center of inertia value (COI reference frame), $\theta_{C O I}$, as the goal is to preserve the system rotor angle stability, which is directly related to these variables [23]. Accordingly:

$$
\begin{aligned}
y(k)-y_{\text {ref }} & =\left[\begin{array}{c}
\theta_{G 1}(k)-\theta_{C O I} \\
\theta_{G 2}(k)-\theta_{C O I}
\end{array}\right] \\
\theta_{C O I} & =\frac{\sum H_{i} \theta_{i}}{\sum H_{i}} \quad \text { with } i=1, \ldots, n_{g}
\end{aligned}
$$

where $n_{g}$ is the number of generators in the system and $H_{i}$ corresponds to the inertia constant of the machine $i$. The cost weights were set as: $Q=I \cdot\left[\begin{array}{lll}0.01 & 0.01\end{array}\right]^{\prime} \mathrm{pu} / \mathrm{deg}^{2}$ and $\quad R=I \cdot\left[\begin{array}{ll}50 & 206\end{array}\right]^{\prime}$ pu (with $P_{d c}^{b a s e}=2.2 \mathrm{GW} \quad$ and $X_{L}^{b a s e}=X_{3}$ ). The green dashed traces in Fig. 7 show the system dynamic response after the outage of Line B with the MPC controller formulated using this cost function. It can be seen that the corrective control preserves the system transient stability (rotor angle responses in Fig. 7 (a) and (b)), thereby allowing higher pre-fault AC boundary transfers (Fig. 7 (c)). The variables manipulated by the MPC controller, $P_{d c}^{*}$ and $X_{L_{A}}^{*}$, are plotted in Fig. 8 in gray solid traces vs. the measured DC power injection $\left(P_{d c}^{\text {meas }}\right)$ and the reactance of TCSC A $\left(X_{L_{A}}\right)$ in green dashed traces. It can be observed that this cost function may lead to the saturation of the actuators, as also seen in [10] when trying to restore the voltage angles to their pre-fault values.
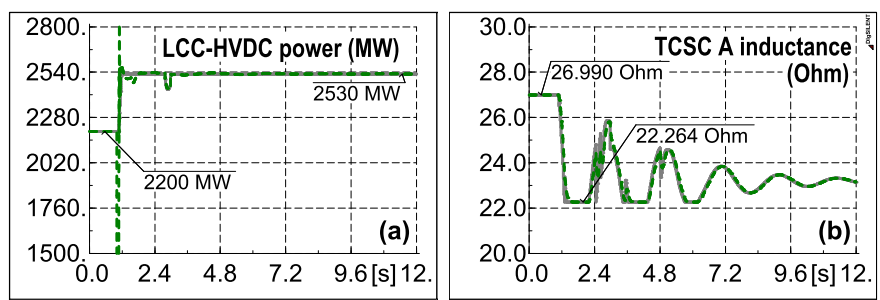

Figure 8. Manipulated variables: (a) LCC-HVDC active power injection: $P_{d c}^{*}$ (gray solid trace) vs. $P_{d c}^{\text {meas }}$ (green dashed trace); (b) TCSC A inductive reactance: $X_{L_{A}}^{*}$ (gray solid trace) vs. $X_{L_{A}}$ (green dashed trace).

\section{Case II. Fixed Rotor Speed Reference Trajectory}

A new objective function considering the deviation of the generators rotor speed from the absolute system frequency, $w_{\text {ref }}=50 \mathrm{~Hz}$, is studied here. As the stability problems directly relate to the speed of the machines, it seems reasonable to try to keep the system frequency close to the nominal operating frequency. The objective function is formulated with these variables:

$$
y(k)-y_{\text {ref }}=\left[\begin{array}{c}
w_{G 1}(k)-w_{\text {ref }} \\
w_{G 2}(k)-w_{\text {ref }} \\
w_{G 3}(k)-w_{\text {ref }}
\end{array}\right]
$$

setting $Q=I \cdot\left[\begin{array}{lll}4 & 4 & 4\end{array}\right]^{\prime} \cdot 10^{3} \mathrm{pu} / \mathrm{Hz}^{2}$ and $R=I \cdot\left[\begin{array}{ll}50 & 206\end{array}\right]^{\prime} \mathrm{pu}$. 
The dynamic response following Line B outage is presented in comparison to the other controller responses with blue dashed traces in Fig. 7. It is clear that the controller not only stabilizes the system, but also manages to improve the system damping, as seen in Fig. 7 (a) and (b) in the generators rotor angles. The controller manipulated variables, shown in Fig. 9, reach a new equilibrium with the LCC-HVDC link carrying $2.41 \mathrm{GW}$ and TCSC A providing $36 \%$ compensation.
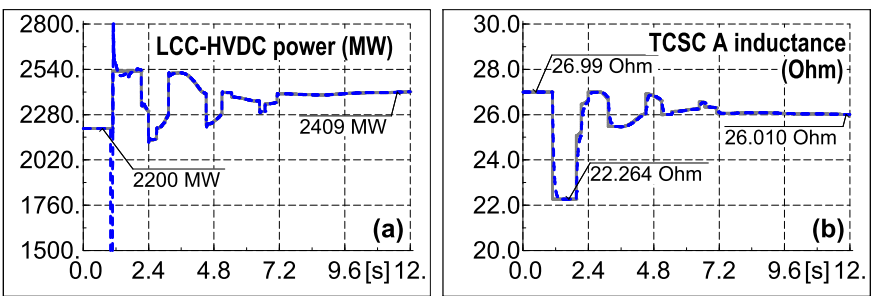

Figure 9. Manipulated variables: (a) LCC-HVDC active power injection: $P_{d c}^{*}$ (gray solid trace) vs. $P_{d c}^{m e a s}$ (blue dashed trace); (b) TCSC A inductive reactance: $X_{L_{A}}^{*}$ (gray solid trace) vs. $X_{L_{A}}$ (blue dashed trace).

\section{Case III. Variable Rotor Speed Reference Trajectory}

Finally, we evaluate a cost function considering a variable reference trajectory. The cost function minimizes the deviation of the generators rotor speed from the average system frequency, also referred as the rotor speed of the center of inertia of the system $\omega_{C O I}(k)[7]$, [9]:

$$
\begin{aligned}
y(k)-y_{\text {ref }}(k) & =\left[\begin{array}{c}
\omega_{G 1}(k)-\omega_{C O I}(k) \\
\omega_{G 2}(k)-\omega_{C O I}(k) \\
\omega_{G 3}(k)-\omega_{C O I}(k)
\end{array}\right] \\
\omega_{C O I}(k) & =\frac{\sum H_{i} w_{i}(k)}{\sum H_{i}} \quad \text { with } i=1, \ldots, n_{g}
\end{aligned}
$$

where $n_{g}$ is again the number of generators in the system and $H_{i}$ the inertia constant of generator $i$. We set as cost function weights: $Q=I \cdot\left[\begin{array}{lll}20 & 20 & 20\end{array}\right]^{\prime} \cdot 10^{3} \mathrm{pu} / \mathrm{Hz}^{2}$ and $R=I \cdot\left[\begin{array}{ll}50 & 206\end{array}\right]^{\prime}$ pu. Alternatively, an analogous generator mean rotor angle trajectory $\theta_{C O I}(k)$, defined in a similar fashion, could have been employed. However, in our experience, it did not produce good results since the monitored rotor angles $\left(\theta_{G 1}, \theta_{G 2}\right)$ vary in the same direction.

Considering again the loss of Line $\mathrm{B}$, the system dynamic response with corrective control in place is shown in light blue solid traces in Fig. 7. With a variable reference trajectory based on rotor speed measurements a good performance is also achieved: the system transient response is improved and a better damping is obtained. The associated change in the manipulated variables is shown in Fig. 10.

The comparison of dynamic responses in Fig. 7 shows that rotor speed measurements (both with respect to a fixed and a variable reference trajectory) are more appropriate signals for the corrective control, providing similar performance for the AC line outages. Overall, the use of corrective control is seen to mitigate the system instability problems, thereby allowing higher pre-fault power transfers across the critical transmission corridor.
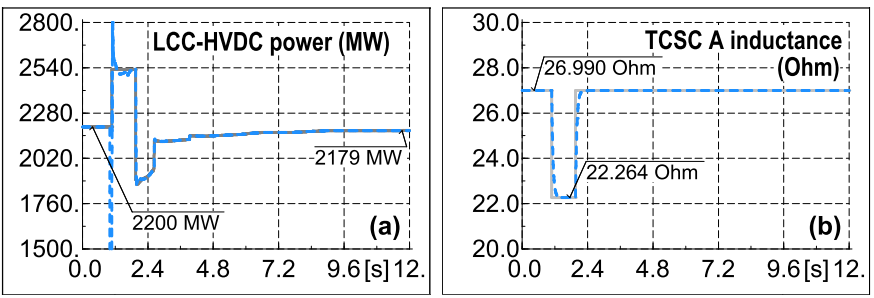

Figure 10. Manipulated variables: (a) LCC-HVDC active power injection: $P_{d c}^{*}$ (gray solid trace) vs. $P_{d c}^{\text {meas }}$ (light blue dashed trace); (b) TCSC A inductive reactance: $X_{L_{A}}^{*}$ (gray solid trace) vs. $X_{L_{A}}$ (light blue dashed trace).

\section{Case Study B: Corrective Control through EASTERN VSC-HVDC}

Corrective control through the Eastern VSC-HVDC link is studied in this section. We employ the three-machine GB equivalent test system, with all the proposed reinforcements, to evaluate the controller performance. The following scenario is analyzed: the Western LCC-HVDC link is operated at a fixed power of $2.2 \mathrm{GW}$ while the Eastern VSC-HVDC carries 1.6 GW. The TCSCs provide $35 \%$ compensation level and each line carries $2.42 \mathrm{GW}$. Again, outage of Line B occurs after a three-phase to ground fault, cleared after $80 \mathrm{~ms}$. Without corrective control in place the system is found to be first swing unstable, as seen in Fig. 11 (a) and (b) in red dashed traces.
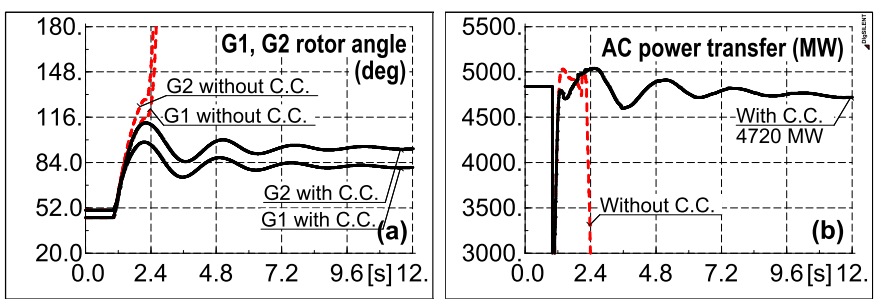

Figure 11. System dynamic response to Line B outage without C.C. (corrective control) in red dashed traces and with C.C. in black traces: (a) rotor angles $\theta_{G 1}, \theta_{G 2}$; (b) total Scotland-England AC boundary power transfer.

We consider now corrective control action through the converters of the VSC-HVDC link. In this case, several control inputs are available: active and reactive power at the rectifier end $\left(P_{\text {rec }}^{*}, Q_{\text {rec }}^{*}\right)$ and reactive power at the inverter end $\left(Q_{i n v}^{*}\right)$ :

$$
u(k)=\left[P_{r e c}^{*}(k) Q_{r e c}^{*}(k) Q_{i n v}^{*}(k)\right]^{T}
$$

We formulate a cost function based on the generators rotor speed to track a constant reference $w_{r e f}=50 \mathrm{~Hz}$, as in (10), with the following input constraints:

$$
\left[\begin{array}{c}
0 \\
-1000 \text { Mvar } \\
-1000 \text { Mvar }
\end{array}\right] \leq\left[\begin{array}{c}
\tilde{P}_{r e c}^{*}(k) \\
\tilde{Q}_{r e c}^{*}(k) \\
\tilde{Q}_{i n v}^{*}(k)
\end{array}\right] \leq\left[\begin{array}{c}
400 \mathrm{MW} \\
1000 \mathrm{Mvar} \\
1000 \mathrm{Mvar}
\end{array}\right]
$$

The limits for the active and reactive power are based on the converter capability limit $P-Q$ curve (due to limitations on the DC cable and DC voltage [24]) and approximated above as a square [9]. Note that the power rating of each converter is 2 GVA $\left(S^{\text {base }}\right)$. The lower limit on the active power was set to avoid power reversal. The application of MPC is interesting 
in this case as it would be preferable to exhaust the headroom available on the $Q$-channels first. Since the $Q$ injections before the fault are close to 0 , there is more headroom on this channel than on the $P$ one which is operating close to the limits of the $P-Q$ curve. The cost weights were set accordingly:

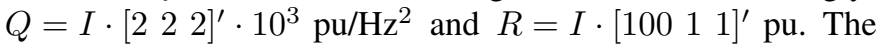
system response with the corrective control is shown in black traces in Fig. 11. The MPC-based controller managed to stabilize the system after the line outage by manipulating the VSC-HVDC link references. The resulting active and reactive power injections are plotted in Fig. 12. An instantaneous violation following the fault clearance is observed for the rectifier active power during and immediately after the fault (Fig. 12 (b)) which can be attributed to the dynamics of the PLL and the inner current controllers of the converter, despite limiting the $P^{*}$ and $Q^{*}$ order. Note that more control effort was exercised through the $Q$ variation, injecting around 250 Mvar after the fault transient (Fig. 12 (c) and (d)) at both ends. The rectifier active power deviation is around $70 \mathrm{MW}$ when the system stabilizes (Fig. 12 (b)).

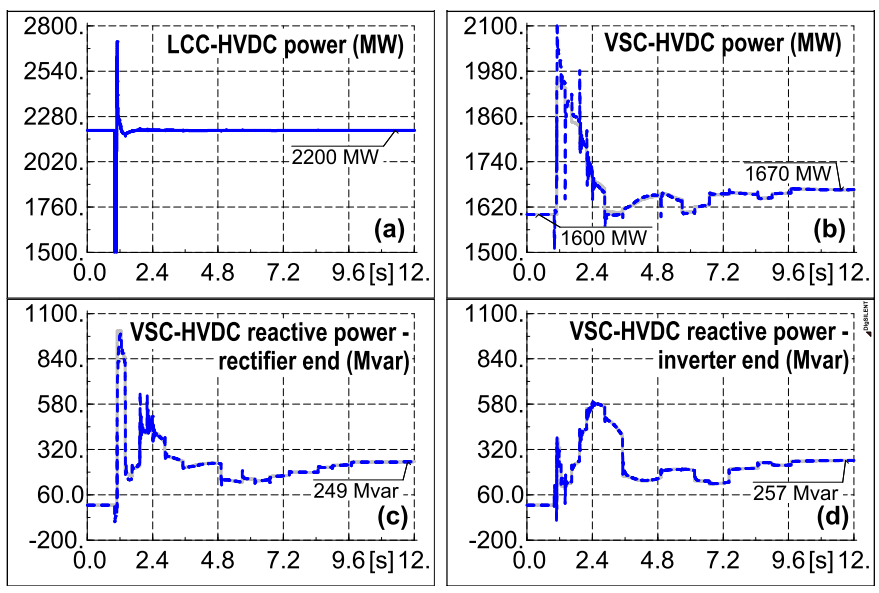

Figure 12. (a) LCC-HVDC active power injection $P_{d c}^{\text {meas }}$. (b) VSC-HVDC rectifier active power injection $P_{r e c}^{*}$ (gray solid trace) vs. $P_{r e c}^{m e a s}$ (blue dashed trace). (c) $Q_{r e c}^{*}$ (gray solid trace) vs. VSC-HVDC rectifier reactive power injection (blue dashed trace). (d) $Q_{i n v}^{*}$ (gray solid trace) vs. VSC-HVDC inverter reactive power injection (blue dashed trace).

\section{CONCLUSION}

The presented results show that the use of MPC algorithm updated with rotor speed wide-area measurements is a promising option for exercising coordinated corrective control though fast power electronic actuators like TCSCs and HVDC links. The corrective control scheme was tested an verified in a three-machine system representative of the GB transmission network to allow higher pre-fault power transfers through the Anglo-Scottish boundary. The proposed methodology could be applied for large-scale systems by obtaining a reduced equivalent model as in (5b)-(5c). The feasibility of the controller implementation (in terms of computation speed of the solution of the QP problem) remains to be checked through real-time simulations, which is out of the scope of this work.

\section{REFERENCES}

[1] (2014) UK future energy scenarios, National Grid. [Online]. Available: http://www2.nationalgrid.com/UK/Industry-information/

[2] (2012) ENSG: Our Electricity Transmission Network: A vision for 2020. [Online]. Available: https://www.gov.uk/government/uploads/ system/uploads/attachment_data/file/48274/4263-ensgFull.pdf

[3] J. Maciejowski, Predictive Control: With Constraints, ser. Pearson Education. Prentice Hall, 2002.

[4] G. Zweigle and V. Venkatasubramanian, "Wide-area optimal control of electric power systems with application to transient stability for higher order contingencies," Power Systems, IEEE Transactions on, vol. 28, no. 3, pp. 2313-2320, Aug 2013

[5] J. Ford, G. Ledwich, and Z. Dong, "Efficient and robust model predictive control for first swing transient stability of power systems using flexible AC transmission systems devices," Generation, Transmission Distribution, IET, vol. 2, no. 5, pp. 731-742, September 2008.

[6] A. Bonfiglio et al., "Improving power grids transient stability via model predictive control," in PSCC 2014, Aug 2014, pp. 1-7.

[7] Y. Phulpin, J. Hazra, and D. Ernst, "Model predictive control of HVDC power flow to improve transient stability in power systems," in SmartGridComm 2011 IEEE, Oct 2011, pp. 593-598.

[8] A. Fuchs, S. Mariethoz, M. Larsson, and M. Morari, "Grid stabilization through VSC-HVDC using wide area measurements," in PowerTech 2011 IEEE Trondheim, June 2011, pp. 1-6.

[9] A. Fuchs, M. Imhof, T. Demiray, and M. Morari, "Stabilization of large power systems using VSC-HVDC and Model Predictive Control," Power Delivery, IEEE Transactions on, vol. 29, no. 1, pp. 480-488, Feb 2014

[10] I. Martínez Sanz, B. Chaudhuri, and G. Strbac, "Corrective control through HVDC links: A case study on GB equivalent system," in PES GM, 2013 IEEE, July 2013, pp. 1-5.

[11] I. Martínez Sanz et al., "Corrective control through Western HVDC link in future Great Britain transmission system," in PES GM, 2015 IEEE, July 2015 , pp. $1-5$.

[12] (2012) NETS Security and Quality of Supply Standard, version 2.2, National Grid. [Online]. Available: http://www2.nationalgrid.com/UK/ Industry-information/Electricity-codes/SQSS/The-SQSS/

[13] S. Achenbach, V. Barry, C. Bayfield, and P. Coventry, "Increasing the GB electricity transmission networks' power transfer capability between North and South; The Western HVDC link," in ACDC 2012, Dec 2012, pp. $1-4$.

[14] O. Anaya-Lara, F. Hughes, N. Jenkins, and G. Strbac, "Influence of wind farms on power system dynamic and transient stability," Wind Engineering, vol. 30, no. 2, pp. 107-127, 2006.

[15] Y. Pipelzadeh, R. Moreno, B. Chaudhuri, G. Strbac, and T. Green, "An assessment of transient assistive measures using HVDC for special protection schemes: Case on the GB transmission system," in $A C D C$ 2012, Dec 2012, pp. 1-6.

[16] N. Hingorani and L. Gyugyi, Understanding FACTS: Concepts and Technology of Flexible AC Transmission Systems. Wiley, 2000.

[17] P. Kundur, Power System Stability And Control, ser. EPRI power system engineering series. McGraw-Hill Education (India) Pvt Limited, 1994

[18] M. Faruque, Y. Zhang, and V. Dinavahi, "Detailed modeling of CIGRE HVDC benchmark system using PSCAD/EMTDC and PSB/SIMULINK," Power Delivery, IEEE Transactions on, vol. 21, no. 1, pp. 378-387, Jan 2006.

[19] A. Yazdani and R. Iravani, Voltage-Sourced Converters in Power Systems: Modeling, Control, and Applications. Wiley, 2010.

[20] M. Bahrman and B. Johnson, "The ABCs of HVDC transmission technologies," Power and Energy Magazine, IEEE, vol. 5, no. 2, pp. 32-44, March 2007.

[21] R. Eriksson and L. Söder, "Wide-area measurement system-based subspace identification for obtaining linear models to centrally coordinate controllable devices," Power Delivery, IEEE Transactions on, vol. 26, no. 2, pp. 988-997, April 2011.

[22] H. Huang, V. Ramaswami, and D. Kumar. Design considerations of ultra high voltage DC system. [Online]. Available: http://www.ptd. siemens.de/050714_Design_Considerations_UHVDC.pdf

[23] G. Zweigle and V. Venkatasubramanian, "Model prediction based transient stability control," in $T \&$ D Conference and Exposition, 2012 IEEE, May 2012, pp. 1-8.

[24] S. G. Johansson, G. Asplund, E. Jansson, and R. Rudervall, "Power system stability benefits with VSC DC-transmission systems," in Cigre Conference 2004 Session, August 2004, pp. 1-8. 